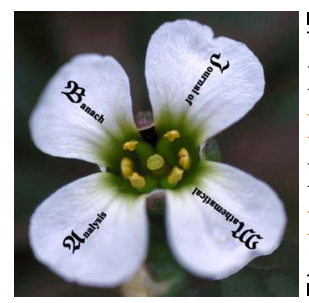

Banach J. Math. Anal. 10 (2016), no. 1, 96-107

http://dx.doi.org/10.1215/17358787-3319442

ISSN: $1735-8787$ (electronic)

http://projecteuclid.org/bjma

\title{
DIFFERENTIABLE FUNCTIONS AND NICE OPERATORS
}

\author{
JUAN CARLOS NAVARRO-PASCUAL* and MIGUEL ÁNGEL NAVARRO \\ Communicated by K. Jarosz
}

\begin{abstract}
The aim of this paper is to describe the operators between spaces of continuously differentiable functions whose adjoint preserves extreme points. It is important to mention that no condition regarding injectivity or surjectivity of the operators is assumed. Previously known results characterizing surjective isometries can be immediately derived from such descriptions.
\end{abstract}

\section{INTRODUCTION AND PRELIMINARIES}

Let $K_{1}$ be a compact Hausdorff space, and let $C\left(K_{1}\right)$ be the space of scalarvalued continuous functions defined on $K_{1}$ endowed with the uniform norm. A well-known result of Arens and Kelley [3] describes the extreme points of the closed unit ball of the dual of $C\left(K_{1}\right)$ as the functions of the form $\alpha \delta_{s}$, where $\alpha$ is a scalar with $|\alpha|=1$ and $\delta_{s}$ is the evaluation functional at an arbitrary point $s \in K_{1}$.

The scalar field $(\mathbb{R}$ or $\mathbb{C})$ is denoted hereafter by $\mathbb{K}$.

If $\mathbb{K}$ is identified with the space of continuous functions defined on a compact Hausdorff space $K_{2}$, reduced to a point, then the mappings $\alpha \delta_{s}$ match the weighted composition operators $T: C\left(K_{1}\right) \rightarrow C\left(K_{2}\right)$ given by

$$
(T f)(t)=e(t) f(\varphi(t)), \quad \text { for any } f \in C\left(K_{1}\right) \text { and } t \in K_{2},
$$

Copyright 2016 by the Tusi Mathematical Research Group.

Received Mar. 21, 2015; Accepted Apr. 15, 2015.

${ }^{*}$ Corresponding author.

2010 Mathematics Subject Classification. Primary 46B20; Secondary 46E15.

Keywords. linear isometry, nice operator, continuously differentiable function space. 


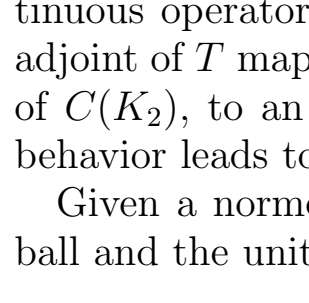

Banach J. Math. Anal. 10 (2016), no. 1, 96-107

http://dx.doi.org/10.1215/17358787-3319442

ISSN: $1735-8787$ (electronic)

http://projecteuclid.org/bjma

\title{
DIFFERENTIABLE FUNCTIONS AND NICE OPERATORS
}

\author{
JUAN CARLOS NAVARRO-PASCUAL* and MIGUEL ÁNGEL NAVARRO \\ Communicated by K. Jarosz
}

\begin{abstract}
The aim of this paper is to describe the operators between spaces of continuously differentiable functions whose adjoint preserves extreme points. It is important to mention that no condition regarding injectivity or surjectivity of the operators is assumed. Previously known results characterizing surjective isometries can be immediately derived from such descriptions.
\end{abstract}

\section{INTRODUCTION AND PRELIMINARIES}

Let $K_{1}$ be a compact Hausdorff space, and let $C\left(K_{1}\right)$ be the space of scalarvalued continuous functions defined on $K_{1}$ endowed with the uniform norm. A well-known result of Arens and Kelley [3] describes the extreme points of the closed unit ball of the dual of $C\left(K_{1}\right)$ as the functions of the form $\alpha \delta_{s}$, where $\alpha$ is a scalar with $|\alpha|=1$ and $\delta_{s}$ is the evaluation functional at an arbitrary point $s \in K_{1}$.

The scalar field $(\mathbb{R}$ or $\mathbb{C})$ is denoted hereafter by $\mathbb{K}$.

If $\mathbb{K}$ is identified with the space of continuous functions defined on a compact Hausdorff space $K_{2}$, reduced to a point, then the mappings $\alpha \delta_{s}$ match the weighted composition operators $T: C\left(K_{1}\right) \rightarrow C\left(K_{2}\right)$ given by

$$
(T f)(t)=e(t) f(\varphi(t)), \quad \text { for any } f \in C\left(K_{1}\right) \text { and } t \in K_{2},
$$

Copyright 2016 by the Tusi Mathematical Research Group.

Received Mar. 21, 2015; Accepted Apr. 15, 2015.

${ }^{*}$ Corresponding author.

2010 Mathematics Subject Classification. Primary 46B20; Secondary 46E15.

Keywords. linear isometry, nice operator, continuously differentiable function space. 


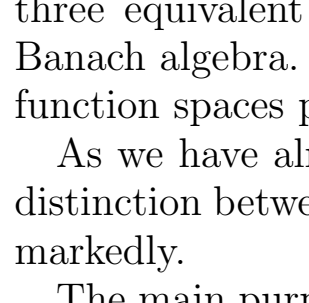

Banach J. Math. Anal. 10 (2016), no. 1, 96-107

http://dx.doi.org/10.1215/17358787-3319442

ISSN: $1735-8787$ (electronic)

http://projecteuclid.org/bjma

\title{
DIFFERENTIABLE FUNCTIONS AND NICE OPERATORS
}

\author{
JUAN CARLOS NAVARRO-PASCUAL* and MIGUEL ÁNGEL NAVARRO \\ Communicated by K. Jarosz
}

\begin{abstract}
The aim of this paper is to describe the operators between spaces of continuously differentiable functions whose adjoint preserves extreme points. It is important to mention that no condition regarding injectivity or surjectivity of the operators is assumed. Previously known results characterizing surjective isometries can be immediately derived from such descriptions.
\end{abstract}

\section{INTRODUCTION AND PRELIMINARIES}

Let $K_{1}$ be a compact Hausdorff space, and let $C\left(K_{1}\right)$ be the space of scalarvalued continuous functions defined on $K_{1}$ endowed with the uniform norm. A well-known result of Arens and Kelley [3] describes the extreme points of the closed unit ball of the dual of $C\left(K_{1}\right)$ as the functions of the form $\alpha \delta_{s}$, where $\alpha$ is a scalar with $|\alpha|=1$ and $\delta_{s}$ is the evaluation functional at an arbitrary point $s \in K_{1}$.

The scalar field $(\mathbb{R}$ or $\mathbb{C})$ is denoted hereafter by $\mathbb{K}$.

If $\mathbb{K}$ is identified with the space of continuous functions defined on a compact Hausdorff space $K_{2}$, reduced to a point, then the mappings $\alpha \delta_{s}$ match the weighted composition operators $T: C\left(K_{1}\right) \rightarrow C\left(K_{2}\right)$ given by

$$
(T f)(t)=e(t) f(\varphi(t)), \quad \text { for any } f \in C\left(K_{1}\right) \text { and } t \in K_{2},
$$

Copyright 2016 by the Tusi Mathematical Research Group.

Received Mar. 21, 2015; Accepted Apr. 15, 2015.

${ }^{*}$ Corresponding author.

2010 Mathematics Subject Classification. Primary 46B20; Secondary 46E15.

Keywords. linear isometry, nice operator, continuously differentiable function space. 


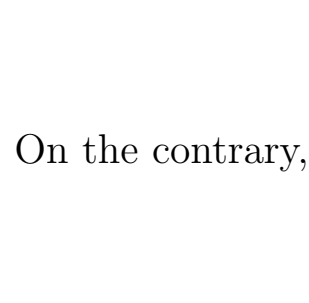

Banach J. Math. Anal. 10 (2016), no. 1, 96-107

http://dx.doi.org/10.1215/17358787-3319442

ISSN: $1735-8787$ (electronic)

http://projecteuclid.org/bjma

\title{
DIFFERENTIABLE FUNCTIONS AND NICE OPERATORS
}

\author{
JUAN CARLOS NAVARRO-PASCUAL* and MIGUEL ÁNGEL NAVARRO \\ Communicated by K. Jarosz
}

\begin{abstract}
The aim of this paper is to describe the operators between spaces of continuously differentiable functions whose adjoint preserves extreme points. It is important to mention that no condition regarding injectivity or surjectivity of the operators is assumed. Previously known results characterizing surjective isometries can be immediately derived from such descriptions.
\end{abstract}

\section{INTRODUCTION AND PRELIMINARIES}

Let $K_{1}$ be a compact Hausdorff space, and let $C\left(K_{1}\right)$ be the space of scalarvalued continuous functions defined on $K_{1}$ endowed with the uniform norm. A well-known result of Arens and Kelley [3] describes the extreme points of the closed unit ball of the dual of $C\left(K_{1}\right)$ as the functions of the form $\alpha \delta_{s}$, where $\alpha$ is a scalar with $|\alpha|=1$ and $\delta_{s}$ is the evaluation functional at an arbitrary point $s \in K_{1}$.

The scalar field $(\mathbb{R}$ or $\mathbb{C})$ is denoted hereafter by $\mathbb{K}$.

If $\mathbb{K}$ is identified with the space of continuous functions defined on a compact Hausdorff space $K_{2}$, reduced to a point, then the mappings $\alpha \delta_{s}$ match the weighted composition operators $T: C\left(K_{1}\right) \rightarrow C\left(K_{2}\right)$ given by

$$
(T f)(t)=e(t) f(\varphi(t)), \quad \text { for any } f \in C\left(K_{1}\right) \text { and } t \in K_{2},
$$

Copyright 2016 by the Tusi Mathematical Research Group.

Received Mar. 21, 2015; Accepted Apr. 15, 2015.

${ }^{*}$ Corresponding author.

2010 Mathematics Subject Classification. Primary 46B20; Secondary 46E15.

Keywords. linear isometry, nice operator, continuously differentiable function space. 


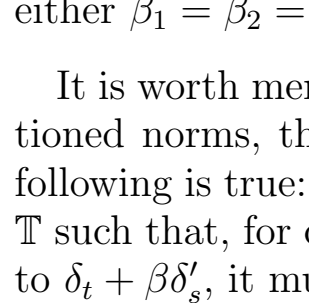

Banach J. Math. Anal. 10 (2016), no. 1, 96-107

http://dx.doi.org/10.1215/17358787-3319442

ISSN: $1735-8787$ (electronic)

http://projecteuclid.org/bjma

\title{
DIFFERENTIABLE FUNCTIONS AND NICE OPERATORS
}

\author{
JUAN CARLOS NAVARRO-PASCUAL* and MIGUEL ÁNGEL NAVARRO \\ Communicated by K. Jarosz
}

\begin{abstract}
The aim of this paper is to describe the operators between spaces of continuously differentiable functions whose adjoint preserves extreme points. It is important to mention that no condition regarding injectivity or surjectivity of the operators is assumed. Previously known results characterizing surjective isometries can be immediately derived from such descriptions.
\end{abstract}

\section{INTRODUCTION AND PRELIMINARIES}

Let $K_{1}$ be a compact Hausdorff space, and let $C\left(K_{1}\right)$ be the space of scalarvalued continuous functions defined on $K_{1}$ endowed with the uniform norm. A well-known result of Arens and Kelley [3] describes the extreme points of the closed unit ball of the dual of $C\left(K_{1}\right)$ as the functions of the form $\alpha \delta_{s}$, where $\alpha$ is a scalar with $|\alpha|=1$ and $\delta_{s}$ is the evaluation functional at an arbitrary point $s \in K_{1}$.

The scalar field $(\mathbb{R}$ or $\mathbb{C})$ is denoted hereafter by $\mathbb{K}$.

If $\mathbb{K}$ is identified with the space of continuous functions defined on a compact Hausdorff space $K_{2}$, reduced to a point, then the mappings $\alpha \delta_{s}$ match the weighted composition operators $T: C\left(K_{1}\right) \rightarrow C\left(K_{2}\right)$ given by

$$
(T f)(t)=e(t) f(\varphi(t)), \quad \text { for any } f \in C\left(K_{1}\right) \text { and } t \in K_{2},
$$

Copyright 2016 by the Tusi Mathematical Research Group.

Received Mar. 21, 2015; Accepted Apr. 15, 2015.

${ }^{*}$ Corresponding author.

2010 Mathematics Subject Classification. Primary 46B20; Secondary 46E15.

Keywords. linear isometry, nice operator, continuously differentiable function space. 


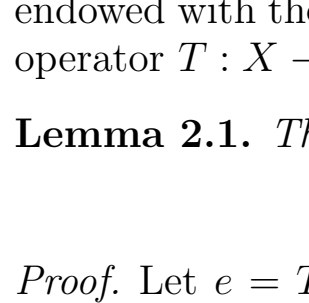

Banach J. Math. Anal. 10 (2016), no. 1, 96-107

http://dx.doi.org/10.1215/17358787-3319442

ISSN: $1735-8787$ (electronic)

http://projecteuclid.org/bjma

\title{
DIFFERENTIABLE FUNCTIONS AND NICE OPERATORS
}

\author{
JUAN CARLOS NAVARRO-PASCUAL* and MIGUEL ÁNGEL NAVARRO \\ Communicated by K. Jarosz
}

\begin{abstract}
The aim of this paper is to describe the operators between spaces of continuously differentiable functions whose adjoint preserves extreme points. It is important to mention that no condition regarding injectivity or surjectivity of the operators is assumed. Previously known results characterizing surjective isometries can be immediately derived from such descriptions.
\end{abstract}

\section{INTRODUCTION AND PRELIMINARIES}

Let $K_{1}$ be a compact Hausdorff space, and let $C\left(K_{1}\right)$ be the space of scalarvalued continuous functions defined on $K_{1}$ endowed with the uniform norm. A well-known result of Arens and Kelley [3] describes the extreme points of the closed unit ball of the dual of $C\left(K_{1}\right)$ as the functions of the form $\alpha \delta_{s}$, where $\alpha$ is a scalar with $|\alpha|=1$ and $\delta_{s}$ is the evaluation functional at an arbitrary point $s \in K_{1}$.

The scalar field $(\mathbb{R}$ or $\mathbb{C})$ is denoted hereafter by $\mathbb{K}$.

If $\mathbb{K}$ is identified with the space of continuous functions defined on a compact Hausdorff space $K_{2}$, reduced to a point, then the mappings $\alpha \delta_{s}$ match the weighted composition operators $T: C\left(K_{1}\right) \rightarrow C\left(K_{2}\right)$ given by

$$
(T f)(t)=e(t) f(\varphi(t)), \quad \text { for any } f \in C\left(K_{1}\right) \text { and } t \in K_{2},
$$

Copyright 2016 by the Tusi Mathematical Research Group.

Received Mar. 21, 2015; Accepted Apr. 15, 2015.

${ }^{*}$ Corresponding author.

2010 Mathematics Subject Classification. Primary 46B20; Secondary 46E15.

Keywords. linear isometry, nice operator, continuously differentiable function space. 


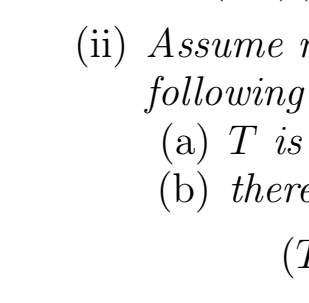

Banach J. Math. Anal. 10 (2016), no. 1, 96-107

http://dx.doi.org/10.1215/17358787-3319442

ISSN: $1735-8787$ (electronic)

http://projecteuclid.org/bjma

\title{
DIFFERENTIABLE FUNCTIONS AND NICE OPERATORS
}

\author{
JUAN CARLOS NAVARRO-PASCUAL* and MIGUEL ÁNGEL NAVARRO \\ Communicated by K. Jarosz
}

\begin{abstract}
The aim of this paper is to describe the operators between spaces of continuously differentiable functions whose adjoint preserves extreme points. It is important to mention that no condition regarding injectivity or surjectivity of the operators is assumed. Previously known results characterizing surjective isometries can be immediately derived from such descriptions.
\end{abstract}

\section{INTRODUCTION AND PRELIMINARIES}

Let $K_{1}$ be a compact Hausdorff space, and let $C\left(K_{1}\right)$ be the space of scalarvalued continuous functions defined on $K_{1}$ endowed with the uniform norm. A well-known result of Arens and Kelley [3] describes the extreme points of the closed unit ball of the dual of $C\left(K_{1}\right)$ as the functions of the form $\alpha \delta_{s}$, where $\alpha$ is a scalar with $|\alpha|=1$ and $\delta_{s}$ is the evaluation functional at an arbitrary point $s \in K_{1}$.

The scalar field $(\mathbb{R}$ or $\mathbb{C})$ is denoted hereafter by $\mathbb{K}$.

If $\mathbb{K}$ is identified with the space of continuous functions defined on a compact Hausdorff space $K_{2}$, reduced to a point, then the mappings $\alpha \delta_{s}$ match the weighted composition operators $T: C\left(K_{1}\right) \rightarrow C\left(K_{2}\right)$ given by

$$
(T f)(t)=e(t) f(\varphi(t)), \quad \text { for any } f \in C\left(K_{1}\right) \text { and } t \in K_{2},
$$

Copyright 2016 by the Tusi Mathematical Research Group.

Received Mar. 21, 2015; Accepted Apr. 15, 2015.

${ }^{*}$ Corresponding author.

2010 Mathematics Subject Classification. Primary 46B20; Secondary 46E15.

Keywords. linear isometry, nice operator, continuously differentiable function space. 


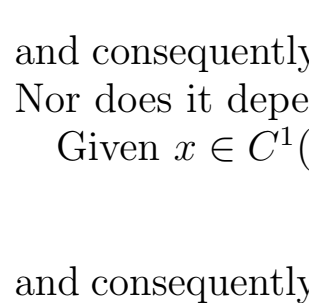

Banach J. Math. Anal. 10 (2016), no. 1, 96-107

http://dx.doi.org/10.1215/17358787-3319442

ISSN: $1735-8787$ (electronic)

http://projecteuclid.org/bjma

\title{
DIFFERENTIABLE FUNCTIONS AND NICE OPERATORS
}

\author{
JUAN CARLOS NAVARRO-PASCUAL* and MIGUEL ÁNGEL NAVARRO \\ Communicated by K. Jarosz
}

\begin{abstract}
The aim of this paper is to describe the operators between spaces of continuously differentiable functions whose adjoint preserves extreme points. It is important to mention that no condition regarding injectivity or surjectivity of the operators is assumed. Previously known results characterizing surjective isometries can be immediately derived from such descriptions.
\end{abstract}

\section{INTRODUCTION AND PRELIMINARIES}

Let $K_{1}$ be a compact Hausdorff space, and let $C\left(K_{1}\right)$ be the space of scalarvalued continuous functions defined on $K_{1}$ endowed with the uniform norm. A well-known result of Arens and Kelley [3] describes the extreme points of the closed unit ball of the dual of $C\left(K_{1}\right)$ as the functions of the form $\alpha \delta_{s}$, where $\alpha$ is a scalar with $|\alpha|=1$ and $\delta_{s}$ is the evaluation functional at an arbitrary point $s \in K_{1}$.

The scalar field $(\mathbb{R}$ or $\mathbb{C})$ is denoted hereafter by $\mathbb{K}$.

If $\mathbb{K}$ is identified with the space of continuous functions defined on a compact Hausdorff space $K_{2}$, reduced to a point, then the mappings $\alpha \delta_{s}$ match the weighted composition operators $T: C\left(K_{1}\right) \rightarrow C\left(K_{2}\right)$ given by

$$
(T f)(t)=e(t) f(\varphi(t)), \quad \text { for any } f \in C\left(K_{1}\right) \text { and } t \in K_{2},
$$

Copyright 2016 by the Tusi Mathematical Research Group.

Received Mar. 21, 2015; Accepted Apr. 15, 2015.

${ }^{*}$ Corresponding author.

2010 Mathematics Subject Classification. Primary 46B20; Secondary 46E15.

Keywords. linear isometry, nice operator, continuously differentiable function space. 


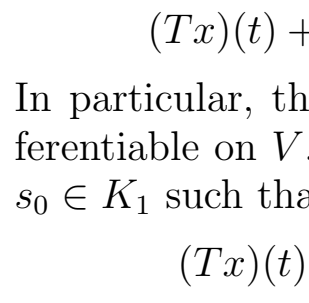

Banach J. Math. Anal. 10 (2016), no. 1, 96-107

http://dx.doi.org/10.1215/17358787-3319442

ISSN: $1735-8787$ (electronic)

http://projecteuclid.org/bjma

\title{
DIFFERENTIABLE FUNCTIONS AND NICE OPERATORS
}

\author{
JUAN CARLOS NAVARRO-PASCUAL* and MIGUEL ÁNGEL NAVARRO \\ Communicated by K. Jarosz
}

\begin{abstract}
The aim of this paper is to describe the operators between spaces of continuously differentiable functions whose adjoint preserves extreme points. It is important to mention that no condition regarding injectivity or surjectivity of the operators is assumed. Previously known results characterizing surjective isometries can be immediately derived from such descriptions.
\end{abstract}

\section{INTRODUCTION AND PRELIMINARIES}

Let $K_{1}$ be a compact Hausdorff space, and let $C\left(K_{1}\right)$ be the space of scalarvalued continuous functions defined on $K_{1}$ endowed with the uniform norm. A well-known result of Arens and Kelley [3] describes the extreme points of the closed unit ball of the dual of $C\left(K_{1}\right)$ as the functions of the form $\alpha \delta_{s}$, where $\alpha$ is a scalar with $|\alpha|=1$ and $\delta_{s}$ is the evaluation functional at an arbitrary point $s \in K_{1}$.

The scalar field $(\mathbb{R}$ or $\mathbb{C})$ is denoted hereafter by $\mathbb{K}$.

If $\mathbb{K}$ is identified with the space of continuous functions defined on a compact Hausdorff space $K_{2}$, reduced to a point, then the mappings $\alpha \delta_{s}$ match the weighted composition operators $T: C\left(K_{1}\right) \rightarrow C\left(K_{2}\right)$ given by

$$
(T f)(t)=e(t) f(\varphi(t)), \quad \text { for any } f \in C\left(K_{1}\right) \text { and } t \in K_{2},
$$

Copyright 2016 by the Tusi Mathematical Research Group.

Received Mar. 21, 2015; Accepted Apr. 15, 2015.

${ }^{*}$ Corresponding author.

2010 Mathematics Subject Classification. Primary 46B20; Secondary 46E15.

Keywords. linear isometry, nice operator, continuously differentiable function space. 


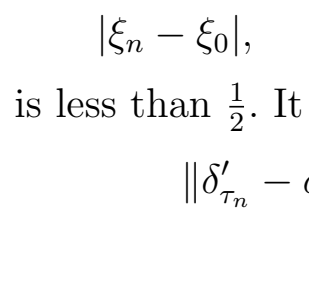

Banach J. Math. Anal. 10 (2016), no. 1, 96-107

http://dx.doi.org/10.1215/17358787-3319442

ISSN: $1735-8787$ (electronic)

http://projecteuclid.org/bjma

\title{
DIFFERENTIABLE FUNCTIONS AND NICE OPERATORS
}

\author{
JUAN CARLOS NAVARRO-PASCUAL* and MIGUEL ÁNGEL NAVARRO \\ Communicated by K. Jarosz
}

\begin{abstract}
The aim of this paper is to describe the operators between spaces of continuously differentiable functions whose adjoint preserves extreme points. It is important to mention that no condition regarding injectivity or surjectivity of the operators is assumed. Previously known results characterizing surjective isometries can be immediately derived from such descriptions.
\end{abstract}

\section{INTRODUCTION AND PRELIMINARIES}

Let $K_{1}$ be a compact Hausdorff space, and let $C\left(K_{1}\right)$ be the space of scalarvalued continuous functions defined on $K_{1}$ endowed with the uniform norm. A well-known result of Arens and Kelley [3] describes the extreme points of the closed unit ball of the dual of $C\left(K_{1}\right)$ as the functions of the form $\alpha \delta_{s}$, where $\alpha$ is a scalar with $|\alpha|=1$ and $\delta_{s}$ is the evaluation functional at an arbitrary point $s \in K_{1}$.

The scalar field $(\mathbb{R}$ or $\mathbb{C})$ is denoted hereafter by $\mathbb{K}$.

If $\mathbb{K}$ is identified with the space of continuous functions defined on a compact Hausdorff space $K_{2}$, reduced to a point, then the mappings $\alpha \delta_{s}$ match the weighted composition operators $T: C\left(K_{1}\right) \rightarrow C\left(K_{2}\right)$ given by

$$
(T f)(t)=e(t) f(\varphi(t)), \quad \text { for any } f \in C\left(K_{1}\right) \text { and } t \in K_{2},
$$

Copyright 2016 by the Tusi Mathematical Research Group.

Received Mar. 21, 2015; Accepted Apr. 15, 2015.

${ }^{*}$ Corresponding author.

2010 Mathematics Subject Classification. Primary 46B20; Secondary 46E15.

Keywords. linear isometry, nice operator, continuously differentiable function space. 


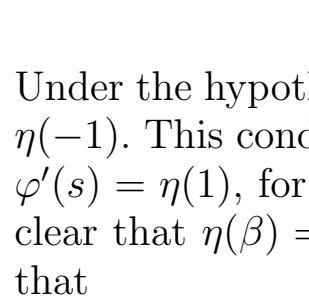

Banach J. Math. Anal. 10 (2016), no. 1, 96-107

http://dx.doi.org/10.1215/17358787-3319442

ISSN: $1735-8787$ (electronic)

http://projecteuclid.org/bjma

\title{
DIFFERENTIABLE FUNCTIONS AND NICE OPERATORS
}

\author{
JUAN CARLOS NAVARRO-PASCUAL* and MIGUEL ÁNGEL NAVARRO \\ Communicated by K. Jarosz
}

\begin{abstract}
The aim of this paper is to describe the operators between spaces of continuously differentiable functions whose adjoint preserves extreme points. It is important to mention that no condition regarding injectivity or surjectivity of the operators is assumed. Previously known results characterizing surjective isometries can be immediately derived from such descriptions.
\end{abstract}

\section{INTRODUCTION AND PRELIMINARIES}

Let $K_{1}$ be a compact Hausdorff space, and let $C\left(K_{1}\right)$ be the space of scalarvalued continuous functions defined on $K_{1}$ endowed with the uniform norm. A well-known result of Arens and Kelley [3] describes the extreme points of the closed unit ball of the dual of $C\left(K_{1}\right)$ as the functions of the form $\alpha \delta_{s}$, where $\alpha$ is a scalar with $|\alpha|=1$ and $\delta_{s}$ is the evaluation functional at an arbitrary point $s \in K_{1}$.

The scalar field $(\mathbb{R}$ or $\mathbb{C})$ is denoted hereafter by $\mathbb{K}$.

If $\mathbb{K}$ is identified with the space of continuous functions defined on a compact Hausdorff space $K_{2}$, reduced to a point, then the mappings $\alpha \delta_{s}$ match the weighted composition operators $T: C\left(K_{1}\right) \rightarrow C\left(K_{2}\right)$ given by

$$
(T f)(t)=e(t) f(\varphi(t)), \quad \text { for any } f \in C\left(K_{1}\right) \text { and } t \in K_{2},
$$

Copyright 2016 by the Tusi Mathematical Research Group.

Received Mar. 21, 2015; Accepted Apr. 15, 2015.

${ }^{*}$ Corresponding author.

2010 Mathematics Subject Classification. Primary 46B20; Secondary 46E15.

Keywords. linear isometry, nice operator, continuously differentiable function space. 


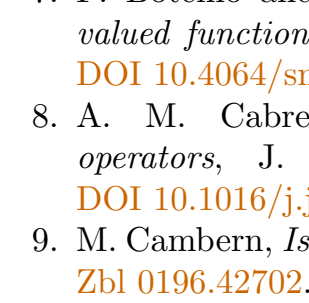

Banach J. Math. Anal. 10 (2016), no. 1, 96-107

http://dx.doi.org/10.1215/17358787-3319442

ISSN: $1735-8787$ (electronic)

http://projecteuclid.org/bjma

\title{
DIFFERENTIABLE FUNCTIONS AND NICE OPERATORS
}

\author{
JUAN CARLOS NAVARRO-PASCUAL* and MIGUEL ÁNGEL NAVARRO \\ Communicated by K. Jarosz
}

\begin{abstract}
The aim of this paper is to describe the operators between spaces of continuously differentiable functions whose adjoint preserves extreme points. It is important to mention that no condition regarding injectivity or surjectivity of the operators is assumed. Previously known results characterizing surjective isometries can be immediately derived from such descriptions.
\end{abstract}

\section{INTRODUCTION AND PRELIMINARIES}

Let $K_{1}$ be a compact Hausdorff space, and let $C\left(K_{1}\right)$ be the space of scalarvalued continuous functions defined on $K_{1}$ endowed with the uniform norm. A well-known result of Arens and Kelley [3] describes the extreme points of the closed unit ball of the dual of $C\left(K_{1}\right)$ as the functions of the form $\alpha \delta_{s}$, where $\alpha$ is a scalar with $|\alpha|=1$ and $\delta_{s}$ is the evaluation functional at an arbitrary point $s \in K_{1}$.

The scalar field $(\mathbb{R}$ or $\mathbb{C})$ is denoted hereafter by $\mathbb{K}$.

If $\mathbb{K}$ is identified with the space of continuous functions defined on a compact Hausdorff space $K_{2}$, reduced to a point, then the mappings $\alpha \delta_{s}$ match the weighted composition operators $T: C\left(K_{1}\right) \rightarrow C\left(K_{2}\right)$ given by

$$
(T f)(t)=e(t) f(\varphi(t)), \quad \text { for any } f \in C\left(K_{1}\right) \text { and } t \in K_{2},
$$

Copyright 2016 by the Tusi Mathematical Research Group.

Received Mar. 21, 2015; Accepted Apr. 15, 2015.

${ }^{*}$ Corresponding author.

2010 Mathematics Subject Classification. Primary 46B20; Secondary 46E15.

Keywords. linear isometry, nice operator, continuously differentiable function space. 